\title{
La educación: clave para el desarrollo humano Una perspectiva desde la educación auténtica
}

\author{
Horacio Ademar Ferreyra*
}

Recepción: 16 de octubre de 2012 • Aprobación: 11 de diciembre de 2012

\section{Resumen}

En este artículo se presentan algunas reflexiones sobre la situación actual de la educación en sus relaciones con lo social, y se señala, desde una perspectiva situada, la importancia que adquieren las humanidades en la formación integral de los sujetos. Para ello, se describe la situación desde una mirada sociohistórica, se revalorizan los aportes significativos y relevantes de las humanidades y se enuncian algunas proyecciones hacia futuro. Asimismo, en este artículo se comparten algunas ideas o principios rectores para un abordaje pleno de la enseñanza y el aprendizaje, en los distintos niveles y modalidades del sistema educativo, especialmente en la educación superior. Esto principios se conciben como alternativa pedagógica y curricular que ayuda a pensar lo educativo en clave del

Doctor y licenciado en Educación (Universidad Católica de Córdoba). Posdoctor en Ciencias Sociales (Universidad Nacional de Córdoba y Universidad Autónoma Metropolitana). Subsecretario de Estado de Promoción de Igualdad y Calidad Educativa del Ministerio de Educación, provincia de Córdoba. Docente e Investigador de la Universidad Católica de Córdoba. Docente e investigador invitado del Doctorado en Educación en la Universidad Santo Tomás, Colombia. Capacitador y consultor educativo en el nivel provincial, nacional e internacional. Autor y coautor de libros, capítulos en libros, artículos, documentos y ensayos relacionados con la temática educativa y escolar. Correo electrónico: hferreyra@ coopmorteros.com.ar 
desarrollo humano. En este marco, y a partir de un diálogo argumentado, se presentan cuestiones relacionadas con el aprender a aprender, aprender a convivir y aprender a emprender, desde la perspectiva de la educación auténtica, concebida no como una teoría, sino como un enfoque integral e integrado, un horizonte de posibilidades que se visualiza como alternativa para superar la fragmentación de los saberes y prácticas en los contextos educativos formales.

Palabras clave: educación social, humanidades, educación integral, enseñanza-aprendizaje, educación superior, desarrollo humano. 


\section{Education: the key to human development A perspective from real education}

\section{Abstract}

This paper presents some reflections on the current situation of education and its relations with the social, and notes, from a situated perspective, the importance that humanities acquire in the comprehensive training of the subjects. For this, the situation is described from a socio-historical perspective; significant and outstanding contributions by the humanities are revalued and some projections into the future are discussed. Also, this paper shares some ideas or guiding principles for a full approach to teaching and learning, at different levels and types of the education system, especially higher education. These principles are designed as a pedagogical and curriculum alternative that helps thinking of education as the key to human development. In this context, and from a valid dialogue, issues related to learning to learn, learning to live and learning to do are presented from the perspective of real education, conceived not as a theory but as a holistic and integrated approach, a horizon of possibilities that is displayed as an alternative to overcome the fragmentation of knowledge and practices in formal educational settings.

Keywords: social education, humanities, comprehensive education, teaching and learning, higher education, human development. 


\section{L'éducation: clé pour le développement humain Une perspective à partir de l'éducation authentique}

\section{Résume}

Dans cet article sont présentées quelques réflexions sur la situation actuelle de l'éducation à propos de ses relations avec le social, et est signalé, à partir d'une perspective située, l'importance des humanités pour la formation intégrale des sujets. Pour cela, la situation est décrite à partir d'un regard sociohistorique, les apports significatifs et d'importance des humanités sont revalorisés et quelques projections vers le futur sont énoncées. Tout de même, dans cet article, sont partagées quelques idées ou principes pour aborder complétement l'enseignement et l'apprentissage, dans les différents niveaux et modalités du système éducatif, notamment en éducation supérieure. Ces principes sont conçus comme une alternative pédagogique et de curriculum qui aide à penser l'éducatif en clé du développement humain. Dans ce contexte, et en partant d'un dialogue argumenté, des questions en relation avec apprendre à apprendre, apprendre à coexister et apprendre à entreprendre se présentent à partir de la perspective de l'éducation authentique, conçue non pas comme une théorie mais comme une approche intégrale et intégrée, un horizon de possibilités qui se rend visible comme alternative pour surmonter la division des savoirs et des pratiques dans les contextes éducatifs formels.

Mots-clés: éducation sociale, humanités, éducation intégrale, enseignement-apprentissage, éducation supérieure, développement humain. 


\section{Los escenarios actuales: preocupaciones, tensiones y deseos}

Hace más de cincuenta años se afirmaba que el mundo se había convertido en una "única y gran sociedad" y que "las contradicciones de la vida" debían entenderse como una "invitación al cambio", mas no como una "enfermedad social y espiritual" (Toynbee, 1949).

Hoy la humanidad percibe esta situación como un "estado de crisis", que lleva en sí misma el potencial del des-orden (desintegración) y la esperanza de un nuevo orden (cambio). Las personas que habitamos el planeta Tierra sufrimos una falta de orientación, no solo respecto de nuestro lugar en el mundo, sino que muchas veces tampoco sabemos qué pensar de estos nuevos tiempos (Lazcano et al., 2001, 2003).

Numerosas son las cuestiones que, en el orden mundial, nacional y local, generan preocupación en la humanidad: las nuevas formas de producción de bienes y servicios; el dinamismo avasallante de la ciencia y la tecnología; la injusta distribución de la riqueza; el desempleo; la pobreza — que en muchos casos se ha convertido en miseria-; los ambientes naturales degradados; las obras y expresiones culturales identitarias en riesgo de desaparición; los modos de consumo que atentan contra la salud integral; las prácticas sociales violentas, discriminatorias, indiferentes y exclusoras que producen desigualdades no solo materiales, sino también simbólicas (por ejemplo, en el acceso a los bienes de la cultura, a las posibilidades de información y al conocimiento que ofrecen las tecnología de la información y la comunicación, a oportunidades de participación en la vida ciudadana y al disfrute pleno de los derechos, entre otras); y el debilitamiento de la democracia y la justicia.

Y también preocupa que en los procesos de toma de decisiones se esté perdiendo la centralidad en lo humano. La democracia liberal se ha convertido en el sistema de legitimación del Estado a nivel mundial, y la economía de mercado, en su correlato económico. En el mundo, y particularmente en América Latina, los Estados-nación están obligados a redefinir la actuación del sector público en el ámbito nacional, subnacional 
y supranacional, a procesar los nuevos conflictos que se generan en el marco de sociedades cada vez más fragmentadas y a promover otras articulaciones con la sociedad civil.

Los problemas actuales no se manifiestan únicamente en las desigualdades de orden político-económico; también las oportunidades de vida son objetivamente distintas, y los valores, expectativas y pertenencias culturales se han tornado diferentes. Estos procesos sociales generan distintos modos de estar en el mundo: modos que encierran nuevas formas de percibir y percibirse, de sentir y sentirse, de comunicar y comunicarse, de interactuar y vivir la ciudadanía.

En el siglo XXI, las sociedades presentan un desarrollo heterogéneo que muestra las dos caras de una misma realidad: globalización y fragmentación, las cuales son debatidas - no sin grandes esfuerzos - para comprender la situación y proyectarse. Esta realidad no permite que sociedades y gobiernos asuman plenamente las transformaciones que se dan en las distintas dimensiones: incorporación de los avances de la ciencia y la tecnología y aplicación de diferentes modelos organizacionales, que derivan de un nuevo orden basado en el conocimiento como herramienta competitiva y la ética como clave para construir una nueva sociedad. Al respecto, Cohen (1998) señala:

Las economías productoras de ideas conocen más desigualdades que aquellas que fabrican objetos. La propensión a excluir a aquellos que no tienen ideas es, según parece, más fuerte que aquella que excluye a quienes carecen de riqueza (Cohen, 1998, pp. 56-57).

En este sentido, sostenemos que las sociedades del conocimiento se van perfilando como una forma superadora de las actuales, pero aún en estado de deseo, porque el conocimiento no es, en muchos lugares del planeta, un bien disponible para todos por igual. 


\section{La educación como política de justicia social: sus aportes al desarrollo humano}

Toda reflexión en torno a las sociedades del conocimiento debiera constituir la ocasión para plantearnos —en consonancia con la Organización de Naciones Unidas (ONU) - un nuevo modelo de desarrollo humano, que no solo se explique desde lo económico, sino que incorpore, entre sus categorías, lo cultural, político, tecnológico, científico, natural —-todas estas dimensiones son constitutivas de una misma realidad - , y que haga posible construir otros escenarios sociales basados en el conocimiento como bien público. Este imperativo supone un renovado desafío en el ámbito de los procesos educativos: encarar las transformaciones ampliando el horizonte a otras dimensiones del desarrollo personal y social, pero no de manera disociada, sino de modo multidimensional, en virtud de que los intereses y necesidades propios de una dimensión requieren de la interacción con las otras.

Le corresponde, entonces, a la educación, como clave para el desarrollo humano, en interacción con las demás esferas societales, constituirse en fuente de oportunidad para construir respuestas situadas en relación con los problemas derivados de los procesos simultáneos $-\mathrm{y}$, a veces, contradictorios - de mundialización, regionalización, democratización, inclusión, polarización, marginación y exclusión. Puede ser este uno de los caminos alternativos que conduzca al logro de un desarrollo humano que les permita a las personas satisfacer sus necesidades (económicas, sociales, de diversidad cultural y de un medio ambiente sano), sin poner en riesgo la satisfacción de estas por parte de las generaciones futuras (ONU, 1987).

En este nuevo escenario, el sistema educativo pasa a ser una prioridad fundamental para la construcción de sociedades más inclusivas y justas, y la educación —concebida como "acción humanizadora" — se asume como una de las herramientas más propicias para contribuir al mejoramiento de la calidad de vida. El filósofo y sociólogo Edgar Morin (1999) define esta necesidad como la necesidad de "salvar al hombre realizándolo" por medio de la educación, como verdadera "política de justicia" 
(Derrida, 1993), respetuosa de la memoria de los que "ya no están o murieron" y de las oportunidades y derechos de las nuevas generaciones que "aún no llegaron o nacieron".

En este marco nos preguntamos: ¿qué educación queremos, aquí y ahora, para la generaciones actuales y futuras, sin olvidar el legado histórico? Y proponemos: una educación que vincule pasado, presente y futuro; que le permita a las personas construir su propio porvenir desde el "respeto por lo "viejo" y la significación de lo "nuevo", y que genere capital intelectual (humano, estructural y relacional) (Román, 2005; Gándara, 2010), en condiciones de calidad y dignidad. En este sentido, Sánchez (2008) indica:

Las nuevas sociedades del conocimiento se construyen teniendo en cuenta las generaciones venideras y la realización de un proyecto común: el desarrollo del hombre y de la humanidad [...] Establecen un nexo entre el progreso del saber, el desarrollo de las tecnologías y las exigencias en materia de participación, dentro de la prospectiva de una ética del futuro (pp. 23-43).

En este sentido, el punto de partida de estas reflexiones es la convicción de que, en cuanto las sociedades del conocimiento se constituyen a partir de una nueva valorización del saber y en el marco de profundas transformaciones inducidas por los avances de la ciencia y la técnica, a la educación le corresponde un lugar central en la evolución de dichas sociedades, particularmente por su incidencia en el desarrollo humano. Pero no cualquier desarrollo, sino un desarrollo humano, sostenible y equitativo para las generaciones presentes y futuras (Programa de las Naciones Unidas para el Desarrollo, 2011).

En este marco adquieren significado y relevancia —en las propuestas formativas de los distintos niveles y modalidades educativas- las humanidades (antropología, lingüística, etnología, teología, historia, artes, literatura, filosofía, religión, comunicaciones, semiótica, etc.) como ciencias que estudian la expresión de la experiencia humana y contribuyen, junto con otras ciencias (formales, sociales y naturales), a la formación integral de la persona. 
El filósofo Ricardo Forster (2008) sostiene:

[En los tiempos actuales] se vuelve imprescindible rescatar esas otras sabidurías esenciales, escondidas, susurradas desde libros olvidados que han sabido marcar a fondo el derrotero de la filosofía y el arte, de la religión y la literatura, de la política y la ética (p. 182).

Esto implica recuperar las metáforas y narrativas mediante las cuales los sujetos y las sociedades se han representado (y se representan) para sí la realidad. En esta convergencia se define, según entendemos, la oportunidad para construir una educación auténtica, es decir, genuina, dinámica, que se da a lo largo de la vida de los sujetos y los hace personas más íntegras y mejores. El punto de partida y de llegada es el hombre como totalidad biopsicosocial y espiritual que piensa, siente y actúa en comunidad.

En el marco de una educación auténtica, las humanidades no son solo una expresión de la experiencia humana, que se transmite de generación en generación, sino que constituyen una oportunidad para transformar la realidad y transformarse a partir de procesos de comprensión inteligente, compromiso ético y construcción situada en torno a la mejora de lo humano. Las humanidades no están ahí como piezas de museo o muestrario de las bellezas del pasado, sino que motorizan nuevas búsquedas y transformaciones en lo personal y social. Frente a los utilitarismos que pretenden colonizar el escenario de lo educativo, puede sostenerse la siguiente necesidad:

\footnotetext{
Un reposicionamiento de la escuela según los fines últimos de la educación: desarrollar, como prioridad, la persona total, sujeto de autonomía y de dignidad, portador de un proyecto único e irrepetible de vida, miembro responsable y participativo en sus comunidades de pertenencia (Carneiro, 2005, p. 21).
}

Este sujeto ha de estar en capacidad de transformar la realidad y no solo de adaptarse a ella (Ferreyra y Peretti, 2006). La educación auténtica no 
entiende lo pedagógico como una cuestión meramente técnica e instrumental, centrada en el aprendizaje individual; por el contrario, rescata la importancia de los procesos de aprendizaje y de enseñanza como instancias de producción dialógica colectiva y de negociación cultural. Supone sujetos - estudiantes, docentes e instituciones-implicados en la situación en la que les toca actuar, que se movilizan por los problemas, que eligen y asumen una posición al respecto y no solo "hacen algo" con ellos, que a partir de las respuestas habituales piensan - a través de una praxis reflexiva- qué otras respuestas son posibles y obran en consecuencia; sujetos que no quedan supeditados a la rigidez de los métodos, sino que se atreven a reemplazarlos por acciones de pensamiento y por nuevas racionalidades.

\section{Desafíos para una educación auténtica: aprendizajes transformadores}

En las últimas décadas del siglo XX e inicios del presente milenio, en la mayoría de los países se han implementado reformas estructurales en el orden institucional-curricular y en la organización de niveles y ciclos, con el propósito de atender los requerimientos de las sociedades del conocimiento. Si bien en todos los casos la mira ha estado en la mejora de los servicios educativos, en términos de igualdad y calidad en el marco de procesos de educación permanente, persiste el desajuste entre lo que la educación ofrece y lo que los protagonistas de sociedades dinámicas y heterogéneas como las actuales esperan de ella. Por otra parte, ciertas particularidades en las formas y el ritmo de aplicación de las reformas han generado, en cada uno de los contextos, consecuencias que hoy requieren de estrategias específicas que permitan superar escollos organizativos, funcionales, pedagógicos y estructurales.

Educar con el objetivo de humanizar en contextos que en ocasiones aparecen desfavorables constituye una ocupación ardua y compleja, y la institución educativa en solitario no puede responder a las múltiples exigencias y renovadas demandas. Hoy la educación es objeto de un intenso 
reclamo por parte de la sociedad y, particularmente, de las familias: un mayor acceso a la educación (principalmente en el nivel medio-secundario y superior) y una formación para la vida. Los sectores de la producción reclaman una formación en competencias que responda a los nuevos requerimientos del mundo del trabajo. Los ámbitos científicos y tecnológicos piden mayor calidad. El Estado manifiesta su preocupación y exige adecuar las prácticas pedagógicas a los tiempos de fluidez que atraviesan nuestras vidas. Ante esto cabe preguntarnos: ¿qué aprendizajes pueden dar respuesta a todos estos requerimientos? ¿Qué es lo que no se puede dejar de enseñar hoy para que los estudiantes puedan comunicarse, trabajar y participar satisfactoriamente en la sociedad?

En este sentido, concordamos con Morin (2005) en la conveniencia de construir una "educación situada", que contextualice los conocimientos sin perder de vista el "todo" (la sociedad global es más que el contexto local), desde una perspectiva que potencie la "conectividad" de los saberes y supere la fragmentación. Es este un camino para enfrentar la "complejidad" de la realidad, desde una perspectiva sociohistórica, para que los "pedazos" de conocimiento que enseñan la universidad o las instituciones educativas no oculten los auténticos problemas de todos los seres humanos. "Hace falta reformar la educación para que la educación transforme las mentalidades" (Morin, 2005, p. 42).

Desde esta visión, consideramos, en consonancia con Tedesco (2003, 2005), que la educación del futuro debería construirse sobre la base de los siguientes tópicos fundamentales: "aprender a aprender" $y$ "aprender a vivir juntos". Nuestra propuesta consistirá en agregar uno más, particularmente significativo: "aprender a emprender", para que todas las personas puedan comunicarse, participar, trabajar en su comunidad y, por ende, vivir en las sociedades del conocimiento (Ferreyra y Peretti, 2006).

El "aprender a aprender" supone dotar a la persona de estrategias para que pueda aprender a conocer (adquirir un repertorio cultural amplio y conocimientos específicos que estimulen la curiosidad de seguir aprendiendo), a hacer (enfrentar situaciones inesperadas, trabajar en equipo, desenvolverse en diferentes contextos sociales y laborales) y a ser 
(conocerse y valorarse a sí mismo, construir la propia identidad y actuar con creciente capacidad de autonomía y de responsabilidad personal en las distintas situaciones de la vida), desarrollando su potencial de aprendizaje durante toda la vida (Delors, 1996). Esta anhelada conjunción del conocer, el hacer y el ser da cuenta de una búsqueda de integridad, paradigma congregador que se opone a la fragmentación de la existencia y a la división mecanicista de la vida.

Sin embargo, con esto no es suficiente. Tal como sostienen Delors (1996) y Tedesco $(2003,2005)$, para habitar como ciudadano en esta sociedad actual, cambiante y diversa se requiere "aprender a convivir", lo cual presupone "aprender a vivir juntos" (incentivar la valoración del otro, percibir las formas de interdependencia y tomar conciencia de la importancia de la comprensión mutua y de la paz, en sociedades cada vez más multiculturales, competitivas y violentas).

En este contexto, consideramos que es menester sumar este tercer elemento: "aprender a emprender", orientado a preparar a los ciudadanos como agentes activos de la transformación de su entorno, a través del desarrollo de actitudes proactivas que, desde el hacer, con saber y conciencia, les posibiliten fijarse metas; formular propuestas; tomar iniciativas; enfrentar con inteligencia, sensibilidad, innovación y creatividad las dificultades, desde sus fortalezas; aprovechar las oportunidades, y superar las amenazas que se le presentan en el escenario actual'.

Esta es, entonces, la educación que proponemos: aquella que ofrezca un currículo significativo y, a la vez, relevante, que promueva la adquisición y el desarrollo de aprendizajes, competencias y capacidades para aprender a aprender, a convivir y a emprender; para ser no solo habitantes, sino ciudadanos plenos de las sociedades del conocimiento.

1 La idea de aprender a emprender en un sentido amplio — más allá de lo económico— ha sido abordada por Ferreyra (1996) en la obra Aprender a emprender, profundizada en el documento "Situación educativa de América Latina y el Caribe: garantizando la educación de calidad para todos" (informe regional de revisión y evaluación del progreso de América Latina y el Caribe hacia la educación para todos, Santiago de Chile) y retomada por el autor en Construir futuros posibles: el desafío de aprender a emprender en las sociedades del conocimiento (2011). 
De esta manera, el desafío principal de la educación está focalizado en la formación de personas que busquen el bien propio y el de los demás; es decir, dueñas de sí mismas, con capacidad para conocer, comprender, emprender, enriquecer y transformar el mundo con su aporte inteligente, sensible y creativo, en cooperación con los demás, durante toda su vida.

Pero para que esto sea posible se requiere no solo del abordaje de los saberes propios de las ciencias formales, sociales y naturales, sino también los de las humanidades, para que las personas puedan transformary transformarse como humanidad. Esto implica el desarrollo de aprendizajes teleológicos o finalísticos (Carneiro, 2005), que impregnen los campos de conocimiento en todos los niveles y modalidades del sistema educativo, complementándose sinérgicamente: saberes sobre la condición humana, su riqueza y diversidad, pero también sobre su contingencia y vulnerabilidad; saberes sobre la ciudadanía, la vida en democracia, los derechos y deberes inalienables; saberes sobre la propia cultura e identidad: lengua, historia, memoria, pensamiento, literatura, artes.

\section{El currículo como catalizador de la realidad: las humanidades en este contexto}

El currículo, en cuanto selección de contenidos, constituye un sistema de construcción de legitimidad cultural en torno a un conjunto de saberes comunes, y expresa acuerdos sobre lo que habrá de enseñarse en el espacio educativo. Adquiere, en consecuencia, un carácter prescriptivo en relación con su función regulatoria como norma escrita. Si bien se registra una apertura de la selección cultural a visiones plurales, así como una participación cada vez más amplia de docentes, académicos, técnicos y representantes de comunidades locales en los procesos de legitimación, es importante considerar que como norma estipula un conjunto de saberes comunes considerados legítimos y valorados por lo que aportan a la transmisión del patrimonio cultural de la humanidad, a la promoción de una mayor justicia social y a la atención al derecho universal a la educación. 
El currículo, como expresión de lo público, constituye una condición de acción política en el contexto de una sociedad democrática. Se constituye, así, en herramienta de la política educativa, porque comunica el tipo de experiencias educativas que son ofrecidas a los estudiantes - consideradas fundamentales para su desarrollo y su participación social-y expresa fundamentalmente el compromiso del Estado como articulador de las diferentes demandas de la sociedad, los estudiantes y las instituciones que los educan (Terigi, 1999). En este sentido, es el Estado el que tiene la capacidad de integrar las diferentes demandas para asegurar la distribución de los bienes culturales con criterio de justicia y afrontando la tensión entre la igualdad de acceso al patrimonio cultural de la humanidad y el respeto por la heterogeneidad de sujetos y grupos sociales y culturales.

Es importante señalar que la construcción pública del contenido de enseñanza - muy especialmente en la educación superior- no es una concepción meramente teórica e ideal, sino que enlaza la teoría y la práctica, el discurso y la realidad, la investigación y la acción; es decir, no solo es currículo lo que se establece a través de documentos, sino también lo que efectivamente se enseña —explícita o implícitamente- - y aprende en las aulas de las instituciones educativas (De Alba, 1995; Connel, 1997; Angulo y Blanco, 2000).

Así, el currículo también es un proyecto político-pedagógico y, por tanto, histórico, social, cultural y educativo, al que le subyacen posiciones ideológicas, sociológicas, psicológicas, epistemológicas y pedagógicas (Schwab, 1974; Gimenos Sacristán, 1981, 1988; Stenhouse, 1984; Coll, 1986; Elliot, 1990; Apple, 1996; Kemmis, 1998; Grundy, 1998). El currículo constituye un marco de actuación profesional para los educadores, y solo en la medida en que sea entendido como herramienta de trabajo, tendrá la capacidad de generar, en cada contexto y en cada institución educativa, un proyecto de acción que haga posible articular la prescripción y las prácticas en términos de enriquecimiento de las experiencias y las trayectorias educativas de los estudiantes.

Acorde con esta intencionalidad, los estados, departamentos (provincias) e instituciones educativas formulan sus proyectos formativos en términos 
de aprendizajes, competencias, capacidades y contenidos que plasman en las diferentes disciplinas, asignaturas, materias, áreas, espacios académicos, etc., y configuran sus estructuras o mallas curriculares. Asimismo, incluyen aspectos pedagógicos didácticos (objetivos, estrategias, recursos, evaluación, entre otros). Las cuestiones epistemológicas, metodológicas y lingüísticas vinculadas con el aprendizaje y la enseñanza adquieren sentido desde una perspectiva situada, contextualizada, para contribuir con el desarrollo de una educación auténtica, en pro del desarrollo humano, pero no cualquier desarrollo, sino un desarrollo humano sostenible en el que se articulen pasado, presente y futuro.

Pensado en estos términos, en la actualidad el currículo debería actuar como un catalizador, con capacidad para contribuir a la transformación de la realidad; esa realidad socioeducativa que no nos ilusiona (Ferreyra, 2006), poniendo el acento en los sujetos, aprendizajes y estrategias que requiere una educación auténtica en los tiempos que corren.

Cuando hablamos de sujetos hacemos referencia a los estudiantes, docentes e instituciones no como meros destinatarios, sino como participantes, considerándolos no desde sus déficits, sino desde la potencialidad para el desarrollo de la aventura educativa. Esto supone cambiar de paradigma y comenzar a ver en los otros no lo que les falta, sino sus potenciales, para formar de manera integral e integrada, para transformar y transformarse.

Pero esto no es suficiente: es necesario pensar en qué aprendizajes debemos abordar en la educación superior - y también, con sus particularidades, en cada uno de los niveles y modalidades del sistema educativo en su conjunto-, porque si no lo hacemos, los sujetos no podrán apropiarse de ellos en otro ámbito que no sea el de las instituciones educativas. Esto nos conduce al para qué y el qué enseñar, en función de potenciar un desarrollo humano sostenible y equitativo en las sociedades del conocimiento. En este sentido, estamos convencidos de que debemos desarrollar un conjunto de competencias genéricas (intelectuales, prácticas, sociales, creativas e interactivas) y específicas que permitan a los sujetos aprender a aprender, a convivir y a emprender. 
Creemos que en este proyecto formativo - tal como lo imaginamos y deseamos- las humanidades juegan un rol decisivo, y queremos aportar algunas ideas en torno a su presencia en la educación superior.

La enseñanza de las humanidades ${ }^{2}$ se realiza, con mayor frecuencia, a partir de la incorporación de asignaturas, materias o espacios específicos vía disciplinaria; por ejemplo, antropología sociocultural, filosofía, expresión artístico-cultural, etc. En las últimas décadas ha emergido un modo de abordaje desde áreas de conocimiento (vía interdisciplinaria integradora); por ejemplo, desde las disciplinas que configuran el área de las ciencias sociales (historia, geografía, sociología, psicología, etc.) o de la comunicación y expresión (lengua y literatura, lengua y cultura extranjera, educación artística y educación corporal); o desde un conjunto de disciplinas, vía multidisciplinaria no interactiva, por ejemplo desde ciencias naturales, tecnología, historia y economía. Por otro lado, constatamos que la vía transdisciplinaria está aún latente en la mayoría de las propuestas formativas de las instituciones, porque siguen primando los territorios de saber epistemológicamente delimitados, por sobre el enfoque integrador de saberes con capacidad para funcionar sistémicamente desde conjuntos problemáticos.

En este punto, y a fin de intentar — como dice Zemelman (2002) - no quedar atrapados en las teorías y evidencias empíricas que muchas veces no nos ayudan a comprender la realidad, proponemos - para no paralizarnos - el abordaje de algunas competencias que consideramos genéricas y, por ello, claves, que si bien pueden ser desarrolladas desde todos los niveles y modalidades educativas, son inexcusables en el ámbito de la educación superior, para potenciar no solo el aprendizaje de las humanidades, sino de todas las ciencias que conforman las propuestas curriculares, a saber ${ }^{3}$ :

2 Se revisaron propuestas formativas de veintidós universidades de Iberoamérica (España, Argentina, Perú, Colombia, México, Chile, Uruguay y Brasil).

3 Construidas a partir del Informe Final del Proyecto Tuning (Beneitone et al., 2007) y la revisión de experiencias de diversos países de Iberoamérica (Argentina, España, Colombia y México). 
Capacidad de abstracción, de análisis y de síntesis

- Capacidad de aplicación de los conocimientos a la práctica

- Capacidad de organización y planificación del tiempo

- Conocimiento sobre el área de estudio y la profesión

- Compromiso ético, social y ciudadano

- Capacidad de comunicarse e interactuar a través de la oralidad, la lectura y la escritura, en la lengua nativa

- Capacidad de comunicarse en una segunda lengua y reconocimiento de la(s) cultura(s) a las que identifica

- Habilidades en el uso de las tecnologías de la información y la comunicación

- Capacidad de investigación y disposición para el aprendizaje permanente, con actitud abierta hacia el cambio y la innovación

- Capacidad para buscar, procesar, analizar y comunicar información

- Pensamiento crítico y capacidad de autocrítica

- Adaptación a nuevas situaciones

- Creatividad

- Capacidad de identificar, abordar y resolver problemas

- Capacidad de toma de decisiones

- Capacidad de trabajo con otros, especialmente en equipos de carácter interdisciplinario

- Capacidad de establecer, sostener y retroalimentar las relaciones interpersonales en pro de metas comunes

- Compromiso activo con la preservación del ambiente y su entorno sociocultural

- Reconocimiento, valoración y respeto de la diversidad multicultural

- Sensibilidad estética y apreciación de las distintas manifestaciones artísticas

- Habilidades para trabajar en un contexto internacional

- Habilidades para el aprendizaje autónomo

- Iniciativa y espíritu emprendedor para la generación de proyectos orientados al desarrollo personal y social

- Compromiso con la calidad 
De esta manera, desde todas las disciplinas científicas y académicas estaríamos contribuyendo a una formación integral e integrada, que permita a los sujetos transformar y transformarse en las sociedades del conocimiento, situadas y conectadas. Así, la educación dejaría de ser mera instrucción, para constituir un proyecto formativo, político y ético cuya meta es un mejor futuro para todos.

Pero también es necesario pensar en las estrategias. Creemos que es pertinente y relevante animarnos a superar -en relación con los aprendizajes en general y el de las humanidades en particular - dos grandes enfermedades que Perkins (2010) enuncia en su libro El aprendizaje pleno: la elementitis y la acerquitis. De acuerdo con el autor, en nuestros sistemas de enseñanza se han encarnado prácticas que poco aportan para un aprendizaje en plenitud; por ejemplo, lo que denomina elementitis, es decir, aprender unidades aisladas y nunca integrarlas en perspectivas generales; y la acerquitis, que es aprender acerca de algo parcialmente, sin llegar a saber hacerlo o mejorarlo. Por ello, en la complejidad actual resulta clave preguntarnos cómo podemos hacer más accesibles los aprendizajes a los estudiantes y qué prácticas educativas podemos proponer para que los aprendizajes sean más estimulantes.

En este sentido, Perkins (2010) propone siete principios del aprendizaje, en el marco de un enfoque que él concibe como una forma distinta de abordar la enseñanza.

1. Actividades holísticas. Estas actividades son las posibilitan un aprendizaje completo. Se trata de buscar versiones "en pequeño" o para principiantes o novatos acerca del desempeño que se quiere aprender. Esto es posible a partir de propuestas basadas en la resolución de problemas, el análisis y discusión de casos, el desarrollo de proyectos. Por ejemplo, si lo que se pretende es el desarrollo de la capacidad de reconocimiento, valoración y respeto de la diversidad multicultural y, al mismo tiempo, el desarrollo de la iniciativa y espíritu emprendedor orientados al aprender a convivir - que suponen la puesta en juego de aprendizajes y contenidos complejos-, podría generarse una propuesta que tome como punto de partida la identificación de situaciones de tensión y conflicto en la 
vida de la propia institución educativa. Igualmente, podrían tomarse algunos casos (que constituirían las "versiones para principiantes") y comprometer a los estudiantes en el análisis de las variables identitarias, socioculturales, ideológicas, políticas, institucionales incidentes; o bien, potenciarse la indagación y desocultamiento de las representaciones que unos sujetos han construido acerca de otros en relación con cuestiones de etnia, religión, pertenencia sociocultural, género, situación económica, orientación política, derechos y obligaciones que suponen los roles de docente y estudiante, entre otras. A partir de allí, el trabajo podría orientarse a la propuesta, desarrollo y gestión de procesos de mediación para la resolución pacífica de conflictos y construcción de consensos, a través del abordaje del conflicto como inherente a la relacionalidad y motor de crecimiento (resolución de problemas).

En esta situación, definida en el micronivel de la institución educativa, podrán anclarse los complejos aprendizajes referidos a los conflictos regionales, nacionales e internacionales (raciales, religiosos, territoriales, económicos, éticos, sociales, etc.) que surgen en los actuales escenarios.

2. Temas estructurantes y temas o tópicos generativos. Esto pretende lograr que valga la pena desarrollar esas actividades, sosteniendo la motivación y el compromiso a través del interés. Esta posibilidad se hace viable si las propuestas de aprendizajes se plantean a partir de temas estructurantes o temas/tópicos generativos (Stone, 1999; Perkins, 1995 y 2010; Perkins y Unger, 2003) que proveen diversidad de conexiones entre nuevos conocimientos y variedad de perspectivas, las cuales, al vincularse con intereses, experiencias y saberes previos de los estudiantes, favorecen el desarrollo de comprensiones complejas y profundas y brindan oportunidades recurrentes para la reflexión y la acción.

3. Trabajar sobre las "partes difíciles". Esto se hace posible mediante la identificación de los nudos problemáticos y al propiciar prácticas sistemáticas que permitan abordar la complejidad. En este marco, la evaluación como proceso resulta imprescindible. 
4. Posibilitar la transferencia. Este principio se materializa mediante el desarrollo de experiencias en contextos diferentes, para favorecer el pensamiento abstracto y la reflexión y también dar lugar a la exploración de diferentes versiones de la actividad o su realización en distintos ámbitos. Para mostrar las posibilidades de articulación del segundo, tercer y cuarto principio, puede tomarse el siguiente ejemplo: patrimonio cultural y memoria colectiva puede ser un tema estructurante o tópico generativo que permita integrar, dinamizar y resignificar aprendizajes propios de la educación artística, en cuanto promueve relaciones entre arte, legado cultural, identidades regionales y tradiciones; de la literatura, en su doble condición de patrimonio cultural en sí misma y transmisora de la herencia cultural de una generación a otra; de las ciencias sociales, en relación con el estudio de las huellas y testimonios de los cambios y continuidades de las sociedades a través del tiempo; de la lengua nativa o de la segunda lengua, considerando las variedades lingüísticas que se hablan en las comunidades de referencia; de la economía, al generar interesantes análisis micro-y macroeconómicos acerca del funcionamiento del mercado de bienes de patrimonio cultural tangible; de la política y el derecho, al estudiar la función reguladora del Estado en la protección, recuperación y conservación del patrimonio nacional; de las ciencias naturales, al abordar temáticas que vinculan estrechamente recursos naturales y patrimonio cultural (zonas protegidas, parques nacionales y reservas marítimas). Por otra parte, la convergencia de miradas de la geografía, la sociología, la economía, la antropología, entre otras, permitiría considerar el caso de aquellas comunidades que viven en zonas protegidas o sus vecindades, al igual que de los programas y proyectos de turismo sostenible que generan oportunidades económicas y benefician a los grupos indígenas y a las mujeres que dependen directamente de la calidad y la disponibilidad de los recursos naturales ${ }^{4}$.

En cada una de las posibilidades enunciadas será posible trabajar las problemáticas implicadas en la conservación del patrimonio cultural, atendiendo a su carácter multidimensional, a través de prácticas diversas

4 Para ampliar, véase: Banco Interamericano de Desarrollo: http://www.iadb.org/es/temas/medioambiente/recursos-naturales-y-patrimonio-cultural,2690.html 
(observación, búsqueda, análisis e interpretación de información de diversas fuentes, trabajos de campo, etc.); esto es, posibilitar la transferencia. Delimitar con precisión los problemas, así como establecer relaciones pertinentes entre las variables culturales, sociales, económicas, ambientales, jurídicas que en ellos interactúan, constituirán tareas realmente complejas del proceso de estudio (las "partes difíciles"). Por eso, el docente deberá anticipar la dificultad e intervenir activamente, trabajar con los estudiantes, aportar los saberes necesarios, ofrecer modelos orientadores.

5. Descubrir lo oculto en las actividades de aprendizaje. Este principio puede resumirse como el hacer explícitas las cuestiones implicadas en cómo aprende el que aprende y cómo enseña el que enseña (estrategias), al igual que reflexionar sobre las relaciones de poder implicadas.

6. Aprender del equipo y de los otros equipos. Esto es, fomentar el trabajo con otros y el aprendizaje por modelos, lo cual requiere del procesamiento grupal de los procesos de interacción e interactividad.

7. Aprender el juego del aprendizaje. Este principio es posible mediante el fomento de la reflexión sobre el propio aprendizaje. Esto supone, en ciertos momentos, como docentes dejar el lugar de conductores, para pasar a ser acompañantes.

Estos tres últimos principios podrían ponerse en juego trabajando, por ejemplo, en proyectos sociocomunitarios o socioocupacionales que, en torno a un ámbito de experiencia (por ejemplo, convivencia intercultural; ambiente social y natural; trabajo decente; vivienda digna; salud, alimentación y adicciones; emprendimientos sociales; tecnologías de la información y la comunicación, etc.), organizaran la enseñanza y el aprendizaje de contenidos propios de una disciplina, asignatura o espacio curricular -o de varios de ellos-, en el marco de espacios formativos de la universidad, diseñados y gestionados por los estudiantes con la orientación y acompañamiento de los docentes (y no con su conducción).

Esto supone la necesidad de poner en marcha procesos de problematización y comprensión de problemáticas de relevancia social 
contemporánea, construir modelos explicativos de las realidades que se aborden, trabajar cooperativamente con otros, tomar decisiones y generar estrategias de acción en el marco de los roles que se hayan asumido en el equipo. El monitoreo y evaluación continuos del propio aprendizaje y el de los demás (y sus múltiples interacciones), el análisis de los logros y dificultades, la reflexión sobre el error y la hipotetización sobre sus causas, la previsión de alternativas y resoluciones posibles, la definición de nuevos rumbos, la búsqueda de nuevos conocimientos para afrontar situaciones constituyen instancias valiosas para la reflexión metacognitiva y el desarrollo de habilidades de autorregulación del aprendizaje. Al mismo tiempo, permiten al docente procesos análogos en su tarea de enseñanza como acompañante y guía de los estudiantes (reflexión sobre el propio aprendizaje, hacer explícitas las cuestiones implicadas en cómo aprende el que aprende y cómo enseña el que enseña y pontenciar las estrategias involucradas en estos procesos).

Para poner en evidencia el modo en que todos estos principios pueden articularse en una misma experiencia educativa, en pro de un aprendizaje pleno en el ámbito de la educación superior, puede recuperarse la estrategia de los semilleros de investigación en Colombia. Sus actividades constituyen una propuesta holística, que permite a los estudiantes experimentar todas las alternativas de los procesos de investigación y adquirir o desarrollar las capacidades involucradas en estos.

Se trata de una valiosa oportunidad para transferir los aprendizajes apropiados en el marco académico formal, resignificándolos mediante su puesta en práctica en distintos contextos, para definir las cuestiones por investigar a partir de los propios intereses, en torno a un tópico generativo o tema estructurante que posibilite abordajes inter- $y$ transdisciplinares. Puede ponerse el siguiente ejemplo: si se considera como tópico generativo el desarrollo tecnológico en la región, los estudiantes podrían investigar - trabajando en equipos - las consecuencias ambientales, los componentes éticos, las transformaciones que han suscitado en las prácticas culturales en general y comunicativas en particular; también podrían abordar o construir información estadística acerca de consumo de tecnología en distintos grupos poblacionales o en distintos territorios, 
abordar el análisis de las transacciones comerciales electrónicas, entre otras posibilidades que muestran la convergencia de saberes de las ciencias naturales, lógico-formales, sociales y humanas.

Las iniciativas de los estudiantes son acompañadas por el docente, quien tiene la responsabilidad de focalizar aquellos aspectos complejos —las "partes difíciles"—, por ejemplo, los procedimientos de formulación de hipótesis, las técnicas de procesamiento y sistematización de la información, las particularidades de los soportes, géneros y lenguajes a través de los cuales es viable comunicar los resultados obtenidos.

Los productos del semillero -en el caso de nuestro ejemplo en relación con el desarrollo tecnológico- podrían presentarse en una feria de investigadores noveles, que constituiría no solo el espacio para mostrar lo realizado, sino para develar (mostrar "lo oculto" del aprendizaje) las estrategias que se pusieron en juego, las capacidades que fueron o no incorporadas en los procesos de aprendizaje y de enseñanza, lo que resulto "fácil" o "difícil", los posibles errores cometidos, lo que se pudo resolver y cómo se lo hizo (reflexión metacognitiva) y dar cuenta de todo ello en un diálogo compartido, que incluirá también el análisis de la acción de acompañamiento y tutoría desarrollada por los docentes.

Sujetos, aprendizajes y estrategias nos invitan a hacer escuela, lo que supone comprender la realidad no solo de manera inteligente, sino también sensible y emprendedora, y a asumir un compromiso ético mediante una construcción socioeducativa de calidad para todos y entre todos.

Parafraseando a Meirieu (2007), no nos podemos contentar con dar de beber a quienes ya tienen sed. También hay que dar sed a quienes no quieren beber. Como el pedagogo señala, se trata de reivindicar el derecho de todos los estudiantes a aprender y la responsabilidad de los profesores y maestros de posibilitarlo. No basta con enseñar, no basta con dar respuesta; hay que provocar en los estudiantes el deseo de aprender (ciencias y humanidades) y de formularse preguntas, de intentar respuestas no solo desde la perspectiva disciplinar, sino también ínter-y transdiciplinaria. Y hacerlo codo con codo, acompañándolos a lo largo 
de toda la trayectoria y ayudándolos a encontrar tiempos de reflexión y concentración en una época de sobreinformación y sobreexcitación en las denominadas sociedades del conocimiento.

\section{Reflexiones finales}

Nuestra propuesta, en síntesis, es proyectarnos hacia una educación superior auténtica, cuyo principio, causa, condición y finalidad sea lo humano y que, en este sentido, procure:

- El abordaje de saberes de las disciplinas que configuran las ciencias humanas y las humanidades (filosofía, artes, literatura, etc.), no solo de las ciencias formales, sociales y naturales.

- El desarrollo de un pensamiento interdisciplinario del saber, que permita articular la cultura científica y la cultura de las humanidades en sus posibilidades de integración.

- La apropiación de conocimientos que permitan a los sujetos ir más allá de la síntesis disciplinaria e interdisciplinaria, para descubrir nuevos fenómenos, problemas y preguntas, para poder contribuir a su resolución desde una perspectiva transdisciplinaria.

- La adquisición y desarrollo de capacidades genéricas y específicas (intelectuales, prácticas, sociales, creativas e interactivas) que permitan a los sujetos formarse integralmente, a fin de poder ser, conocer, actuar, crear, convivir y emprender en las sociedades del conocimiento.

- El fortalecimiento de la dignidad humana, la valoración de las diferencias, el respeto mutuo, el compromiso ético hacia un mundo social caracterizado por la convivencia intercultural y la paz.

Estas aspiraciones implican un cambio significativo en el nivel de las instituciones educativas; un cambio de orientación, de mirada, de presupuestos metodológicos y de proyectos de trabajo que aseguren la apropiación de conocimientos sobre los asuntos que preocupan y ocupan a los individuos y a las comunidades, como dispositivo de interpretación de la realidad. Se trata, entonces, de formular propuestas que, desde la 
reflexión, permitan a los estudiantes cuestionar visiones y situaciones naturalizadas y conciliar comprensión, juicio crítico, acción creadora y transformadora. Se trata, también, de construir espacios formativos innovadores, dinámicos y cooperativos, en los que los estudiantes se sientan implicados y asuman protagonismo, en el marco de propuestas que, al tiempo que conectan con sus propias inquietudes cognitivas, personales, afectivas, culturales, políticas, sociales, les permitan una apertura respetuosa a las inquietudes, necesidades, modos de pensar, ser y vivir de los otros. Esas propuestas deben habilitar la posibilidad de que los sujetos en formación desempeñen diversos roles en un trabajo comprometido con la calidad y la ética.

Se hace necesario, entonces, en el ámbito de la educación superior, superar la inteligencia láser que, en su especialización, pasa por alto las influencias recíprocas, para potenciar el desarrollo de una inteligencia faro, que profundiza en las realidades que aborda, examina sus interacciones con el contexto, establece conexiones, enfrenta la complejidad (Gardner, 2005).

Nos convocamos a comprometernos en un proyecto formativo atento a los temas significativos, que propicie un aprendizaje expansivo impulsado por la creatividad y la curiosidad, que habilite comprensiones profundas que permitan, siempre, ver más allá de lo aprendido (Perkins, 2012). Nos convocamos, en suma, a avanzar hacia una educación de calidad, que no solo sea eficiente y eficaz, sino también pertinente, relevante y equitativa en el marco de un desarrollo humano sostenible para todas y todos.

\section{$\mathbf{R}_{\text {eferencias }}$}

Angulo, J. F. y Blanco, N. (coords.) (2000). Teoría y desarrollo del currículum. Málaga: Aljibe.

Apple, M. (1996). El conocimiento oficial. La educación democrática en una era conservadora. Barcelona: Paidós. 
Baquero Lazcano, P. et al. (2001-2003). La mundialización en la realidad argentina (tomos I y II). Córdoba: El Copista.

Beneitone, P., Esquetini, C., González, J., Marty, M., Siufi, G. y Wagenaar, R. (2007). Reflexiones y perspectivas de la educación superior en América Latina. Informe final del Proyecto Tuning América Latina 2004-2007. Bilbao, España: Universidad de Deusto Universidad de Groningen. Recuperado de http:// tuning.unideusto.org/tuningal/index.php?option=com_docman\&ltemid= 191\&task $=$ docclick\&bid $=54 \&$ limitstart $=0$ \&limit $=5$

Carneiro, P. (2005). La educación, el aprendizaje y el sentido. Presentación en el Encuentro Sentidos de la Educación y la Cultura. Cultivar la Humanidad. Santiago: Oficina Regional de Educación de la Unesco para América Latina y el Caribe.

Cohen, D. (1998). Riqueza del mundo, pobreza de las naciones. México: Fondo de Cultura Económica.

Coll, C. (1986). Los niveles de concreción del diseño curricular. Cuadernos de Pedagogía, 139, 23-30.

Connell, R. W. (1997). Escuelas y justicia social. Madrid: Morata.

De Alba, A. (1992). Evaluación curricular. Conformación conceptual del campo. México: Universidad Nacional Autónoma de México.

Delors, J. (1996). Informe: La educación encierra un tesoro. Madrid: Santillana -Unesco.

Derrida, J. (1993). Los spectres de Marx. París: Galilée.

Elliot, J. (1990). La investigación-acción en educación. Madrid: Morata.

Ferreyra, H. (1996). Aprender a emprender. Buenos Aires: Novedades Educativas. 
Ferreyra, H. (2006). Transformación de la educación media en la Argentina. Tensiones y conflictos en el diseño e implementación en la provincia de Córdoba. Córdoba: Universidad Católica de Córdoba.

Ferreyra, H. (2011). Construir futuros posibles: el desafío de aprender a emprender en las sociedades del conocimiento. Tiempo de Educar, 12(23), 9-28. Recuperado de http://redalyc.uaemex.mx/src/inicio/ArtPdfRed. jsp?iCve=31121090002

Ferreyra, H. y Peretti, G. (comps.) (2006). Diseño y gestión de una educación auténtica. Buenos Aires: Novedades Educativas.

Forster, R. (2008). El laberinto de las voces argentinas. Buenos Aires: Colihue.

Gándara, G. (2010). Salvaguarda de los saberes para el mundo del trabajo y su relación con las competencias laborales. En M. R. Almandoz et al. Educación y trabajo: articulaciones y políticas. Buenos Aires: Unesco.

Gardner, H. (2005). Las cinco mentes del futuro. Un ensayo educativo. Barcelona: Paidós.

Gimeno Sacristán, J. (1981). Teorías de la enseñanza y desarrollo del currículum. Madrid: Anaya.

Gimeno Sacristán, J. (1988). El currículum: una reflexión sobre la práctica. Madrid: Morata.

Grundy, S. (1998). Producto o praxis del currículum. Madrid: Morata.

Kemmis, S. (1998). El currículum: más allá de la teoría de la reproducción. Madrid: Morata.

Meirieu, P. (2007). Es responsabilidad del educador provocar el deseo de aprender (entrevista). Cuadernos de Pedagogía, 373, 42-47. 
Morin, E. (1999). Los siete saberes necesarios para la educación del futuro. París: Unesco-Santillana.

Morin, E. (2005). Repensar la reforma, reformar el pensamiento (entrevista). Cuadernos de Pedagogía, 342, 42-46. Recuperado de http://www.humanizarleyendo.cl/recursos/entrevistas/morin.pdf

Orealc y Unesco (2007). Situación educativa de América Latina y el Caribe: garantizando la educación de calidad para todos. Informe regional de revisión y evaluación del progreso de América Latina y el Caribe hacia la EPT. Santiago de Chile: EPT/PRELAC. Recuperado de http://unesdoc.unesco.org/ images/0015/001528/152894S.pdf

Organización de Naciones Unidas (1987). Our common future: Brundtland Report. Recuperado de http://www.un-documents.net/our-common-future.pdf

Perkins, D. (1995). La escuela inteligente: del adiestramiento de la memoria a la educación de la mente. Barcelona: Gedisa.

Perkins, D. (2010). El aprendizaje pleno. Principios de la enseñanza para transformar la educación. Buenos Aires: Paidós.

Perkins, D. (2012, septiembre). Enseñar para comprender en el siglo XXI. Conferencia. II Simposio Internacional de Enseñanza para la Comprensión en Educación Superior. Córdoba: Universidad Nacional de Villa María.

Perkins, D. y Unger, C. (2003). Andes. Proyecto Enseñanza para la Comprensión de la Escuela de Educación del Posgrado de Harvard. Harvard University, Grupo de Habilidades Cognitivas, Project Zero, Harvard Graduate School of Education, Cambridge.

PNUD (2011). Sostenibilidad y equidad: un mejor futuro para todos. Informe sobre Desarrollo Humano 2011. Nueva York. 
Román, N. (2005). Capital intelectual: generador de éxito en las empresas. Visión Gerencial, 3(2), 67-79. Recuperado de http://www.saber.ula.ve/bitstream/123456789/25076/2/articulo6.pdf

Sánchez Burlón, J. M. (2008). La infancia en la sociedad del conocimiento. Revista CTS, 4(11), 23-43. Recuperado de http://oeibolivia.org/files/Volumen\%20 4\%20-\%20N\%C3\%BAmero\%2011/art02.pdf

Schwab, J. (1974). Un enfoque práctico para la planificación del currículo. Buenos Aires: El Ateneo.

Stenhouse, L. (1984). Investigación y desarrollo del currículum. Madrid: Morata.

Stone Wiske, M. (1999). La enseñanza para la Comprensión. Vinculación entre la investigación y la práctica. Buenos Aires: Paidós.

Tedesco, J. C. (2003). Los pilares de la educación del futuro. Buenos Aires: IIPEUnesco. Recuperado de http://www.iipebuenosaires.org.ar/system/files/ documentos/pilares-educacion-futuro.pdf

Tedesco, J. C. (comp.) (2005). Educar en la sociedad del conocimiento. Buenos Aires: Fondo de Cultura Económica.

Terigi, F. (1999). Currículum. Itinerarios para aprender un territorio. Buenos Aires: Santillana.

Toynbee, A. J. (1949). La civilización puesta a prueba. Buenos Aires: Emecé.

Zemelman, H. (2002). Pensar teórico y pensar epistémico: los retos de las ciencias sociales latinoamericanas. México: Instituto Pensamiento y Cultura en America. Recuperado de http://images.sociologia07.multiply.multiplycontent.com/attachment/0/RoMqTgoKCpkAAE5BcEg1/Doc\%20ZEMELMAN. doc?nmid $=47728633$ 